\title{
IN VITRO RELEASE OF HYDROCORTISONE BY GLYCINE-IMMOBILIZED EVAL MEMBRANE
}

\author{
Ping-Shan LaI ${ }^{1}$, TAI-Horng Young ${ }^{1}$, Cheng-Yi WAnG ${ }^{1,2}$, Ming-Jium ShIEH ${ }^{1,3}$ \\ ${ }^{1}$ Institute of Biomedical Engineering, National Taiwan University \\ ${ }^{2}$ Department of Internal Medicine, National Taiwan University Hospital \\ ${ }^{3}$ Department of Oncology, National Taiwan University Hospital \\ Taipei, Taiwan
}

\begin{abstract}
A pH-sensitive membrane for colon-specific drug delivery was prepared by glycineimmobilization on poly (ethylene-co-vinyl alcohol) (Gly-EVAL) that can enhance the permeability of hydrocortisone at $\mathrm{pH} 7.4$ and resist drug permeation at pH 2.0 or in gastric juice. As the results of drug releasing profile, it is proposed that the electrical repulsion occurring between adjacent carboxylate ions at pH 7.4 on Gly-EVAL causes the higher permeation rate of hydrocortisone. Consequently, the hydrocortisone coated by Gly-EVAL can escape from degradation in acid environment and release significantly in neutral or weak basic $p H$ values, which is ideally suitable for local treatment of ulcerative colitis.
\end{abstract}

Biomed Eng Appl Basis Comm, 2005 (April); 17: 27-30.

Keywords: EVAL membranes, $\mathrm{pH}$-sensitive, hydrocortisone.

\section{INTRODUCTION}

Ulcerative colitis (UC) is a relapsing disease of the colonic mucosa with unknown cause characterized by bloody diarrhea. Traditional treatment of UC was rested on the use of sulfasalazine and either topical or systemic steroid. However, the use of sulfasalazine has been limited by its side effects that are thought to be mainly related to the sulfa portion of the molecule. Recent studies have also proved that 5-amoinosalicylic acid (5-ASA) is the active moiety in sulfasalazine which acts tropically in the lumen of the intestine. Therapy usually involves 5-ASA, which are of limited benefit, with a response rate between 30 and 80

Received: Feb 18, 2005; Accepted: Mar 3, 2005

Correspondence: Ming-Jium Shieh M.D., Ph.D.

Department of Oncology, National Taiwan University Hospital, Taipei, Taiwan

E-mail: soloman@ha.mc.ntu.edu.tw percent, depending on the end point used [1], or corticosteroids such as prednisolone, although resistance and dependence can become problematic with corticosteroids [2]. Consequently, attention has thus focused on the preparation of pure 5-ASA and prednisolone either as a retention enema, suppository or slow-releasing tablet.

Within recent years, the development of drug delivery systems capable of selective release of drug in the colon has attracted much attention [3-5]. Several approaches utilized in achieving colon targeting include use of $\mathrm{pH}$-sensitive polymer coatings [6], time-dependent formulations [7], bacterial degradable coatings [8-12], biodegradable polymer matrices and hydrogels [13-14] and prodrugs [15-18]. To date, many low molecular and macromolecular prodrugs are ready to be utilized for colonic delivery.

Since there is growing interest in the specific delivery of drugs to the colon for local treatment of $\mathrm{UC}$, we design $\mathrm{pH}$-sensitive membranes for colon-specific drug delivery synthesized by the modification of poly (ethylene-co-vinyl alcohol) 
(EVAL) membranes. The membranes are functioning as protective carriers of drugs in the stomach and small intestine. It is believed that the impact of gastric juice on the polymer membranes is mainly due to the low $\mathrm{pH}$ value of the gastric acid. Beside gastric acid, however, there are pepsin, mucus, and bacteria in the gastric juice. Therefore, the aim of this study is to design a glycine-immobilized EVAL membrane for hydrocortisone release, and to investigate the effect of gastric juice on the polymer membranes applied in the controlled release.

\section{EXPERIMENTAL}

\subsection{Materials}

EVAL (E105A, containing ca. 56mole\% vinyl alcohol) was kindly supplied by Kuraray Co. (Japan) and used as received. Ethanol was purchased from Showa Chemical (Japan). Water was double distilled and deionized before use. In this study, EVAL was dissolved in the ethanol-water cosolvent to prepare membranes. Analytic-grade hexamethylene diisocyanate (HMDI, TCI, Japan), 4dimethylaminopyridine (DMAP, Lancaster, England), N,N-dimethylformamide (DMF, Fisher, U.S.A.), disuccinimidyl oxalate (DSO, Fluka, Switzerland), glycine (Lancaster, England), and acetone (Tedia, U.S.A.) were used to modify the surface of EVAL membranes to result in the conversion of the membrane to highly $\mathrm{pH}$-sensitive. Hydrocortisone was purchased from Sigma (U.S.A.) and used as the model drug for diffusion study. Gastric juice was obtained by suction from volunteer patients during endoscopy when they underwent routine physical checkup. Oral informed consent was obtained in advance.

\subsection{Membrane Preparation}

EVAL was dissolved in co-solvent containing 40 vol. \% water and 60 vol. \% ethanol to form 15 wt. \% EVAL homogeneous solution at $60^{\circ} \mathrm{C}$. This solution was dispersed uniformly on a glass plate (ca. $120 \mu \mathrm{m}$ ) at $60^{\circ} \mathrm{C}$, and then was immediately placed in an air-circulated oven at $60^{\circ} \mathrm{C}$ until the casting solution became a solid membrane. The thickness of the membrane was $16 \pm 1 \mu \mathrm{m}$.

\subsection{Surface Modification}

Immobilization of glycine on the EVAL membrane and chemical characteristics was carried out in the following procedure as reported previously [5]. In Brief, the EVAL membrane was immersed in HMDI $(10 \% \mathrm{v} / \mathrm{v})$ in the presence of DMAP $(0.5 \% \mathrm{w} / \mathrm{v})$ for $4 \mathrm{hr}$ for isocynation of hydroxyl group of membrane surface. The isocyanated EVAL membrane was further immersed in $1 \mathrm{~N}$ aqueous $\mathrm{NaOH}$ solution to hydrolyze the surface isocyanate group. Subsequently, the amino group was converted to the activated ester in DMF containing a prescribed amount of DSO for an hour. The glycine coupling reaction was performed in an aqueous solution of prescribed amount of glycine at pH 9 for an hour, which formed urethane bonds between glycine and aminoalkylated EVAL membrane. After the immobilization, the glycine-immobilized EVAL membrane (Gly-EVAL) was sufficiently washed with water for removal of adsorbed glycine.

\subsection{Membrane Surface Morphologies}

The morphologies of the membranes were examined by a scanning electron microscope (SEM). The freeze-dried samples were gold coated and viewed with SEM (S-800, Hitachi, Japan) at $20 \mathrm{kV}$. The surface morphologies of the membranes were also characterized by tapping mode atomic force microscopy (AFM, DIGITAL INFTRUMENTF Multimotestm 5597E).

\subsection{Attenuated Total Reflection / Fourier Transform Infrared Spectroscopy}

The Gly-EVAL membranes were analyzed by attenuated total reflection (ATR) / Fourier transform infrared (FTIR) spectroscopy with a Nicolet Impact 410 spectrophotometer provided with an ATR device. All spectra were taken by 40 scans at a nominal resolution of $4 \mathrm{~cm}^{-1}$.

\subsection{Permeability of Hydrocortisone}

Permeation by diffusion of hydrocortisone through the prepared membranes was studied at 37 $\mathrm{aC}$ in gastric juice, and in buffer solutions with $\mathrm{pH} 2.0$ and $\mathrm{pH}$ 7.4, respectively. The diffusion experiments were carried out in a two-chamber, well-stirred diffusion cell with a volume of $40 \mathrm{ml}$ each. The membrane was placed between two chambers with 5.3 $\mathrm{cm}^{2}$ of available membrane area. The stirring speed in each chamber was maintained at approximate $600 \mathrm{rpm}$ using independently controlled motors. Since the permeability measurement was performed under sufficient stirring, the diffusion resistance at the liquidmembrane interface was neglected.

One of the diffusion chambers was filled with gastric juice or buffer solution containing hydrocortisone with an initial concentration of $50 \mu$ $\mathrm{g} / \mathrm{ml}$ and the other chamber was filled with buffer solution or gastric juice only. The permeability of hydrocortisone through the EVAL and Gly-EVAL membranes was monitored by an UV spectrophotometer (Ultrospec 1000E, Pharmacia 
Biotech, Sweden) from the peak absorbance at $242 \mathrm{~nm}$. Each experiment was repeated four times and the results were expressed as the mean of the four results.

\section{RESULTS AND DISCUSSION}

\subsection{Membrane Morphology}

Macroscopically, the EVAL membrane and the Gly-EVAL membrane appeared transparent. The EVAL membrane appeared fairly dense structure with few holes existing in the membrane (data not shown). After surface modification, the Gly-EVAL membrane still showed similar dense morphology (Fig. 1). Thus, the processes of surface modification would not change the membrane structure under the observable detection sensitivity of SEM. Fig. 2 shows the characteristics of the membrane surface by AFM. As shown in Fig. 2a, the EVAL membrane surface was smooth, the Gly-EVAL membrane surface, however, was nano-scale uneven that might be due to the glycine immobilization on EVAL membrane (Fig. 2b).

\subsection{Characterization the Surface Modification}

The surface modification was also analyzed by ATR-FTIR, which enabled us to determine the chemical composition of EVAL membrane surface by a significant new peak at $1682 \mathrm{~cm}^{-1}$, which could be attributed to the formation of $\mathrm{C}=\mathrm{O}$ bond (data not shown). The amount of glycine immobilized on the EVAL membrane was quantitatively determined by measuring the difference of the absorbance of the solution at $280 \mathrm{~nm}$ between the initial and the final glycine concentrations.

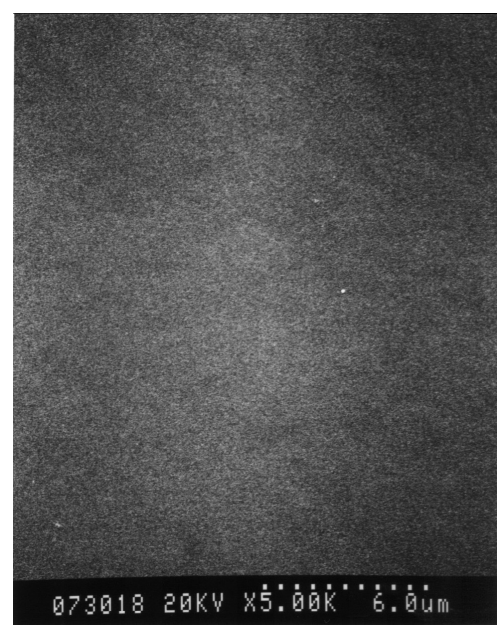

Fig 1. SEM photomicrographs of a Gly-EVAL membrane.

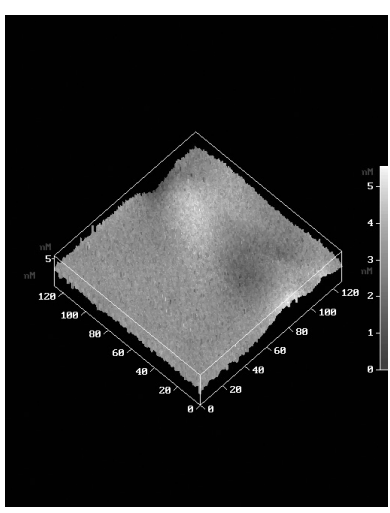

2(a)

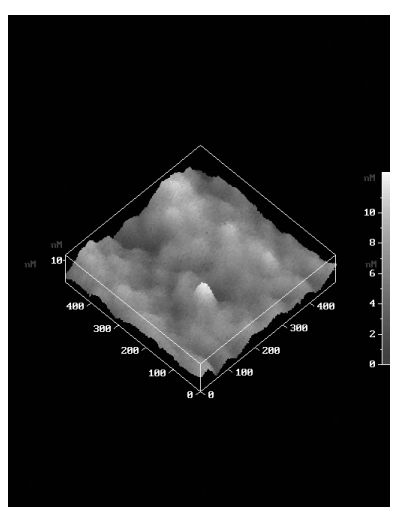

2(b)
Fig 2. AFM photomicrographs of EVAL (a) and Gly-EVAL (b) membranes.

\subsection{Hydrocortisone Permeability}

Fig. 3 shows the time dependence of the cumulative amount of hydrocortisone permeation through EVAL membrane in gastric juice and buffer solutions with different $\mathrm{pH}$ values at $37^{\circ} \mathrm{C}$. It is obvious that the cumulative amount of hydrocortisone in the receptor side at $\mathrm{pH} 7.4$ was high. Furthermore, the permeation rate of hydrocortisone through EVAL membrane in gastric juice was conspicuously small. It is believed that the dense membrane structure led to the low permeation rate of hydrocortisone and the effects of substances contained in the gastric juice, such as pepsin, mucus, and etc., blocked the path of drug permeation. Consequently, though the $\mathrm{pH}$ values were similar, the permeation rate of hydrocortisone was relatively higher in $\mathrm{pH} 2.0$ buffers than that in gastric juice.

Time course permeation by diffusion to hydrocortisone through Gly-EVAL membrane was also studied with the same procedures (Fig. 4). As the unmodified EVAL membrane data, the cumulative amount of hydrocortisone permeated across the GlyEVAL membrane appeared to be virtually the same in the acid buffer solution. Furthermore, because of the low hydrocortisone permeation rate the dense structure of the Gly-EVAL membrane seemed intact in gastric juice, which meets the requirement of colon-specific drug delivery system which is able to prevent the coated drug from degradation in the acidic environment. In contrast, the permeation rate of hydrocortisone through the Gly-EVAL membrane was much higher in the buffer solution of $\mathrm{pH} 7.4$ compared to that of $\mathrm{pH} 2.0$ and in gastric juice. The cumulative hydrocortisone amount through the Gly-EVAL membrane was higher than that through the EVAL 


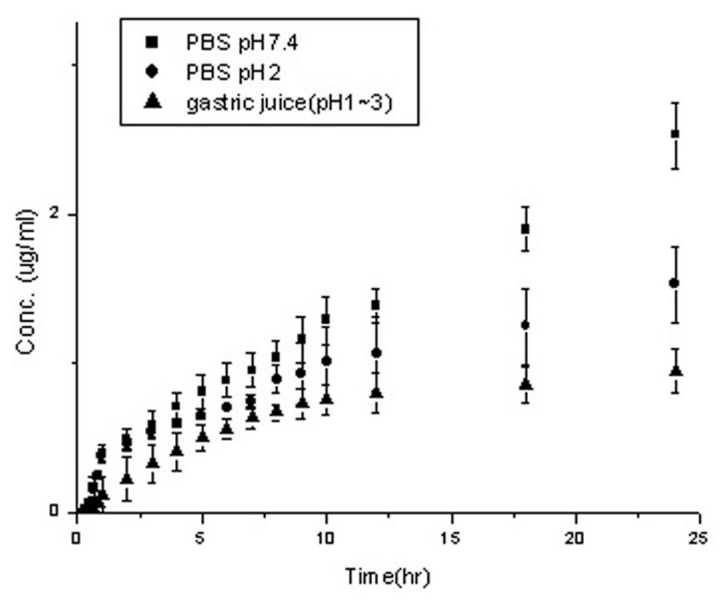

Fig 3. Cumulative amount of hydrocortisone permeation through EVAL membranes in the buffer solutions of $\mathrm{pH} 7.4, \mathrm{pH} 2.0$ and in gastric juice.

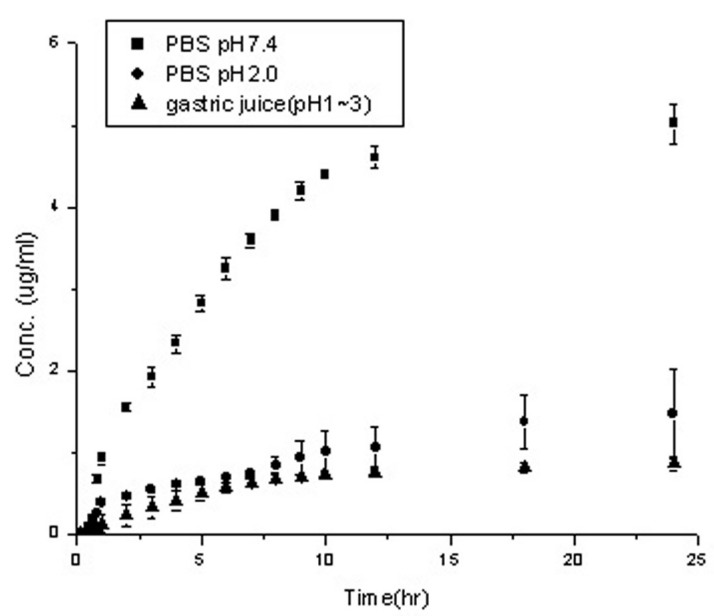

Fig 4. Cumulative amount of hydrocortisone permeation through Gly-EVAL membranes in the buffer solutions of $\mathrm{pH} 7.4, \mathrm{pH} 2.0$ and in gastric juice.

membrane after $24 \mathrm{~h}$. It is suggested that the electrical repulsion occurs between adjacent carboxylate ions leading to a transition of compact coiled polymer chains to extend and swell themselves. The equilibrium swelling of EVAL and Gly-EVAL membranes shows the same trend at both $\mathrm{pH} 2.0$ and $\mathrm{pH} 7.4$ [5]. This indicates that the Gly-EVAL membrane can provide different permeability for hydrocortisone in different $\mathrm{pH}$ environment according to the dissociation mechanism of the carboxyl groups of glycine immobilized on EVAL membrane.
In conclusion, the electrical repulsive force occurred between the ionized carboxyl groups at $\mathrm{pH}$ 7.4 on Gly-EVAL membrane that caused the higher permeation rate of hydrocortisone. By glycine immobilization, the nearly $\mathrm{pH}$-insensitive EVAL membrane transformed to $\mathrm{pH}$-sensitive EVAL membrane, and the dense membrane structure showed no obvious change in gastric juice. Therefore, the GlyEVAL membrane could be further applied in colonspecific drug delivery.

\section{ACKNOWLEDGEMENTS}

This research was supported by the fund provided by National Taiwan University Hospital (NTUHS-901000-25).

\section{REFERENCES}

1. Hanauer SB. Medical therapy of ulcerative colitis. Lancet 1993; 342:412-7.

2. Farrell RJ and Peppercorn MA. Ulcerative colitis. Lancet 2002; 359:331-40.

3. Mrsny RJ, The colon as a site for drug delivery, J. Control. Rel., 1992; 22:15-34.

4. Watts PJ and Illum L, Colonic Drug delivery, Drug Dev. Ind. Pharma., 1997; 23:893-913.

5. Shieh MJ, Lai PS and Young TH. 5-Aminosalicylic acid permeability enhancement by a $\mathrm{pH}$-sensitive EVAL membrane. J. Membr. Sci. 2002;204:237-246.

6. Akala EO, Kopeckova P and Kopecek J, Novel pHsensitive hydrogels with adjustable swelling kinetics, Biomaterials, 1998; 19:1037-1047.

7. Gazanica A, Sangali ME and Giordano M, Oral chronotopic drug delivery systems: Achievement of time and/or site specificity, Eur. J. Pharm. Biopharm., 1994; 40:246-250.

8. Saffran M, Field JB, Pena J, Jones RH and Okada Y, Oral insulin in diabetic dogs, J. Endocrinol., 1997; 13:267-278.

9. Mooter GVD, Samyn C and Kinget R, Azo polymers for colon-specific drug delivery, Int. J. Pharm., 1992; 87:37-46.

10. Mooter GVD, Samyn C and Kinget R, Azo polymers for colon-specific drug delivery. Part II: Influence of the type of azo polymer on the degradation by the intestinal microflora, Int. J. Pharm., 1993; 97:133-139.

11. Milojevic S, Newton JM, Cummings J, Gibson GR, Bothman RL, Ring SG, Allwood MC and Stockham M, Amylose, the new perspective in oral drug delivery to the human large intestine, STP Pharma Sci., 1995; 5:47-53. 
12. Kalala W, Kinget R, Mooter GVD and Samyn C, Colonic drug-targeting: in vitro release of ibuprofen from capsules coated with poly(etherester) azopolymers, Int. J. Pharm., 1996; 139:187195.

13. Rubinstein A, Radai R, Ezna M and Pathnack S, In vitro evaluation of calcium pectate: a potential colon-specific drug delivery, Pharm. Res., 1993; 10:258-263.

14. Bronsted H, Hovgaard I and Simonsen I, Dextran hydrogels for colon-specific drug delivery IV. Comparative release study of hydrocortisone and prednisolone phosphate, STP Pharma Sci. 1995; 5:65-69.
15. Kopecek J, Kopeckova P, Bronsted H, Rathi R, Rihova B, Yeh PY and Ikesue K, Polymers for colon specific drug delivery, J. Control. Release, 1992; 19:121-130.

16. Jung YJ, Lee JS and Kim YM, Synthesis and in vitro / in vivo evaluation of 5-aminosalicyl-glycine as colon-specific pro drug of 5-aminosalicylic acid, J. Pharm. Sci., 2000; 89:594-602.

17. Kopecek J, The potential of water-soluble polymeric carriers in targeted and site-specific drug delivery, J. Control. Release, 1990; 11:279-290.

18. Sakuma S, Lu ZR, Kopeckova P and Kopecek J, Biorecognizable HPMA copolymer-drug conjugates for colon-specific delivery of 9aminocamptothecin, J. Control. Release, 2001; 75:365-379. 